\title{
Experimental approach to the recognition of truthful and false mental responses based on the wavelet transform of the electroencephalogram
}

\author{
Yumatov EA ${ }^{1 *}$, Potapov VYu ${ }^{2}$, Karatygin NA ${ }^{1}$, Dudnik EN ${ }^{1}$ and Pertsov SS ${ }^{1,3}$ \\ ${ }^{1} \mathrm{PK}$ Anokhin Research Institute of Normal Physiology, Moscow, Russia \\ ${ }^{2} \mathrm{AS}$ «Interfax», Russia \\ ${ }^{3}$ Moscow State University of Medicine and Dentistry Named after IA Evdokimov, Moscow, Russia
}

\begin{abstract}
An existing polygraph technique to evaluate the faithfulness of the verbal response is based on recoding of psychophysiological and somatic-and-autonomic indices, which do not reflect the actual deceitful or truthful state of the subject's brain.

The advanced method of electroencephalogram wavelet transform was developed in recent years. This approach allowed us to determine a principle possibility for the direct, objective recording of mental activity in the human brain.

The overall goal of the research is to develop a fundamentally new information technology to identify a truthful and deceitful state in the brain mental activity, which suggests the wavelet transform of the electroencephalogram and machine learning.

For this purpose, an experimental model and software have been created and described in the article for recognizing the truthful and false mental responses of a person based on the electroencephalogram analysis.

The software was developed with Microsoft Visual Studio 2017 and Net Framework 4.5. This software is simple in use and works with the Russian language interface.

The developed experimental model and information-software allow us to compare electroencephalographic indicators of two mental states of brain activity, one of which is deceitful, and the other is truthful.
\end{abstract}

\section{Introduction}

A polygraph, or lie detector, is extensively used in criminal investigations and vocational selection for the testing of false and truthful answers of a person. This technical equipment serves for the conduction of instrumental psychophysiological studies [1-3].

Polygraph testing has a serious disadvantage. The result is evaluated from recording of psychophysiological indices, which only indirectly reflect the individual subjective state and do not serve as the criteria for a real mental activity (i.e., deceitful or truthful state of the subject's brain).

In some countries, including Germany, Poland and USA, the results of psychophysiological examination are not considered as evidence.

The limitations of a polygraph study are associated with the fact that this method does not suggest direct recording of mental activity of the human brain, which reflects its deceitful or truthful state.

The brain represents a unique organization in the living nature that is capable of mental activity. This is manifested in consciousness, feelings, and emotions, i.e., in the subjective self-perception and perception of the outside world. However, the nature of brain mental activity remains unclear.

A variety of researchers, including I.P. Pavlov, P.K. Anokhin, N.P. Bekhtereva, A.M. Ivanitskii, K.V. Sudakov, T. Nagel, K. Popper, R.W.
Sperry, F. Crick and C. Koch pointed out that it is necessary to study the nature of brain mental activity [4-12].

Actually, the existing methods of psychophysiology and neurophysiology do not allow direct recording and studying the patterns of brain mental activity (thoughts, emotions, and feelings). The mechanisms for these phenomena are beyond objective scientific researches [13-15]. This fact contributes to the "chasm" between an understating of mental and neurophysiological events in the brain (as pointed out by T. Nagel) [8].

Our researches allowed scientists to approach understating of brain mental activity [13-15].

The advanced methods of electroencephalogram (EEG) wavelet transform were developed in recent years [16-19].

The EEG wavelet transform allowed us to determine a principle possibility for the direct, objective recording of mental activity in the

${ }^{*}$ Correspondence to: Yumatov EA, PK Anokhin Research Institute of Normal Physiology, Moscow, Russia, E-mail: eayumatov@mail.ru

Key words: information technologies, electroencephalogram (EEG), EEG wavelet transform, brain mental activity, consciousness, polygraph, truth and falseness

Received: May 21, 2019; Accepted: July 01, 2019; Published: July 04, 2019 
human brain [20-21]. These data hold much promise to develop a new information technology for the recognition of truthful and false states of the human brain.

This work was designed to develop a fundamentally new information technology to identify a truthful and deceitful state in the brain mental activity, which suggests the EEG wavelet transform.

For this purpose, it is necessary to develop an experimental model and software for recognizing the truthful and false mental state of the human brain.

\section{Description of the experimental model}

The PC-based experimental device has two monitors, which are simultaneously used during testing of mental responses from a subject.

The fist monitor is intended for an experimenter, which makes settings in the main window of Control Panel (Figure 1).

The window of Control Panel consists of several parts.

1. The left upper part includes the list of questions and images that will be demonstrated to a subject. This part also has the drop-down list, where an experimenter specifies the test result (truth or falseness).

2. The right upper part includes a panel with experimental settings and buttons to control the course of observations.

3. The left lower part includes the window with a journal of observations to get the text information in testing.

4. The right lower part includes an auto-updated copy of the subject's monitor (i.e., an experimenter can control the monitor view of a test person).
The second monitor is intended for a test person. The monitor window appears as a single-color display. An image with the text of question (Figure 2) or only question (in the absence of image) successively appears on this screen.

A special device was constructed to synchronize the mental response of a test subject with the EEG recording. This device includes a light sensor, which is put on the experimenter's monitor screen opposite to the Signal Window (SW). The device with this sensor is connected to the input of an electroencephalograph channel.

SW is represented by a light or black square, which alternate each other in testing (Figure 3)

SW is black-colored in an inactive state. Therefore, the light sensor does not react to this object. SW on the experimenter's monitor screen is colored white, when some image with a question disappears from the monitor screen of a test person. Such changes are followed by signaling of the light sensor, which appears as a mark point on the EEG recording.

This software allows us to conduct observations in the automatic or semiautomatic mode.

In the automatic mode, our software performs certain manipulations for each question after the start of testing (according to the algorithm that is specified in the Testing Program window). By the end of this algorithm, the program passes to the next question. So the algorithm is repeated for this question.

In the semiautomatic mode, the software makes a pause and "waits" for pushing the button by an experimenter to pass to the next question.

In the beginning of each testing, the image and question appear on the monitor screen of a test person. The time of demonstration can

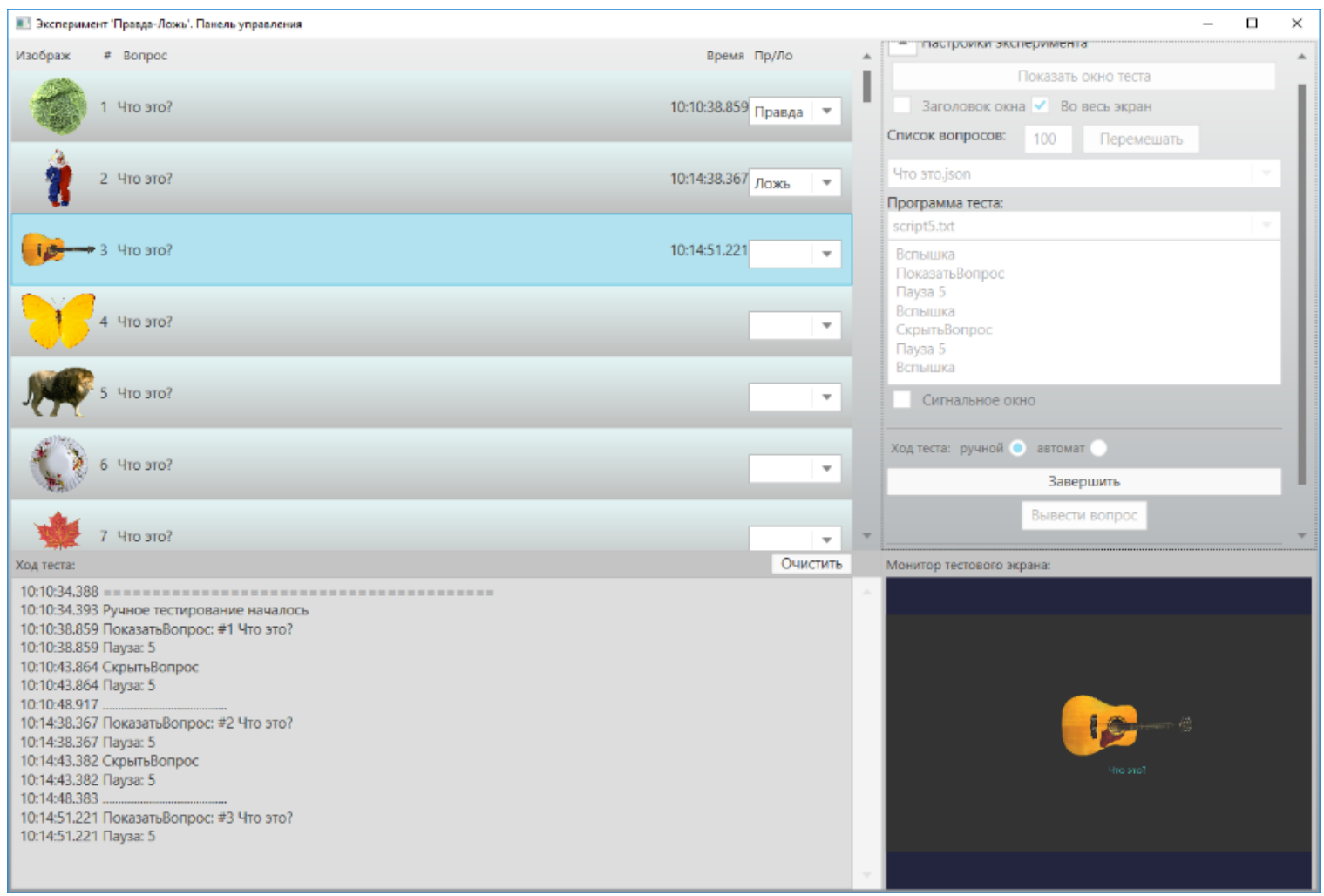

Figure 1. Main window of the monitor for an experimenter 


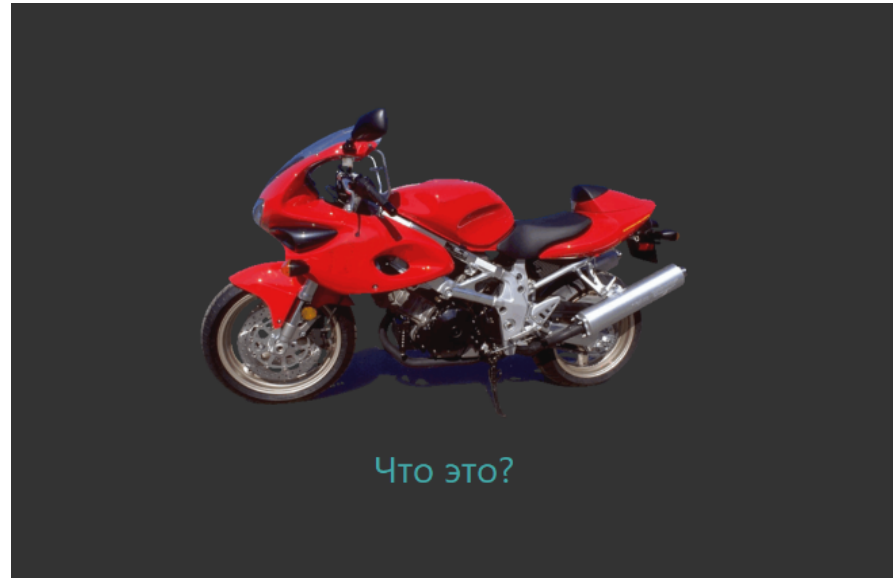

Figure 2. The exposure window of the monitor for a test person

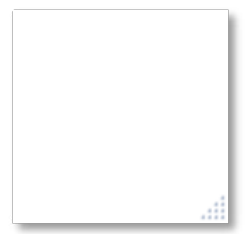

1

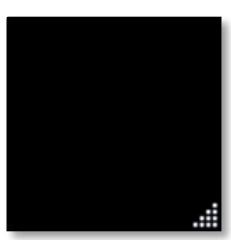

2
Figure 3. View of the signal window: 1 -active state, 2 -inactive state

vary from 1 to $10 \mathrm{sec}$ with software. This duration is set to $5 \mathrm{sec}$ in the standard settings. During this period, SW of the experimenter's monitor screen is black-colored.

Immediately after the disappearance of an image from the subjects' monitor screen, he must give a truthful or false mental response (according to the instruction). The time for this response can be adjusted from 1 to $10 \mathrm{sec}$ with software. This period is set to $5 \mathrm{sec}$ in the standard settings.

When an image disappears from the subjects' monitor screen, the color of SW on the experimenter's monitor screen immediately changes to white. The light sensor reacts to these changes, and a signal mark is transmitted to the EEG channel to synchronize the EEG recording with the mental response of a test person.

The software allows us to select randomly a set of images, which consecutively appear on the screen (every time). The number and sequence of questions can be varied with settings of Control Panel. Moreover, it is possible to change some parameters of the subject's monitor screen (window color; and size and color of font). These settings can be found in the file of software settings.

During or by the end of observations, an experimenter can evaluate the result of each test ("Truth" or "Falseness") using a drop-down list for the question.

By the end of observations, the results can be stored in CSV file for a further processing (e.g., with Excel software).

The file consists of lines, which includes the following parameters for each question:

- Time of question (with an accuracy to milliseconds),

- Order number of question,

- Text of question,
- Name of image, and

- Result of test (Truth or Falseness).

This software has the database, which includes 180 pictures with identifiable visual impressions (e.g., animals, plants, household items, furniture, and transport) and questions. The images have a sufficiently high resolution (300 dpi) and are in color. Each image is colorful and sharp.

The software can be installed on PC under Windows 7, 8, or 10 with two monitors: first one for an experimenter, and the second one for a test person.

Our software was developed with Microsoft Visual Studio 2017 and Net Framework 4.5. This software is simple in use and works with the Russian language interface (Figs. 1 and 2) [22].

EEG is recorded during testing for a further comparative wavelet analysis of results in two mental states of the subject's brain (false and truthful).

The testing of mental responses акщь a test person proceeds in several stages.

Before the start of this procedure, EEG electrodes are fixed on the head surface of a test person in various leads.

An experimenter provides instructions to a test person. Some image and question will appear on the monitor screen in front of a test person, who knows exactly the correct answer. The duration of imageand-question representation can be set by an experimenter (average time $5 \mathrm{sec}$ ).

When this image disappears from the monitor, a test person gives the truthful or false mental response. The time for response is set by an experimenter (average time $5 \mathrm{sec}$ ).

Depending on the question, the mental response can be short ("Yes" or "No") or comprehensive. For example, for the presentation of an elephant picture and asking the question "What is it", the response can be truthful ("This is the elephant") or false ("This is the fly").

EEG is recorded during the mental response. After this procedure, a test person is asked about the truth or falseness of his response. The fact of a truthful or false mental response is entered in the protocol. Then all the files with EEG recordings during the mental response will be analyzed by the wavelet transform and machine learning [16-21].

The developed experimental model and information-software allow us to evaluate and compare EEG indicators of two mental states of brain activity, one of which is deceitful, and the other is truthful.

\section{Acknowledgements}

This work was supported by the Russian Foundation for Basic Research (project No. 19-07-00008/19, "Development of an information technology for the recognition of truthful and false thought responses in the brain mental activity by means of electroencephalogram analysis with the wavelet transform and machine learning").

\section{References}

1. Kholodnyy Yu I (1999) Analysis of Physiological Reactions during Examination with a polygraph: Practical guide. Moscow, pp: 6-52.

2. Ogloblin SI, Molchanov A (2004) Instrumental "lie detection": Academic Course Yaroslavl': Nyuans, pp: 464. 
3. Zhurin SI (2011) Practice and theory of using the lie detectors. 2nd Edition, Stereotype. Moscow: Goryachaya Liniya - Telekom, pp: 144

4. Pavlov IP (1951) Twenty-year experience of objective studying the higher nervous activity (behavior) of animals. Complete set of works. Moscow - Leningrad: Izd. AN SSSR.

5. Anokhin PK (1969) Mental form of reality reflection. Collection "Leninskaya theory of reflection and today's world. Pavlov T, Part 1, Chapter 3, pp: 109.

6. Bekhtereva NP, Budzen PV, Gogolitsyn Yu L (1977) Brain codes of mental activity. Leningrad: Nauka, pp: 165.

7. Ivanitskiy AM (1999) Main mystery of the nature: How do subjective feelings form on the basis of brain processes? Psikhologicheskiy Zhurnal 20: 93-104.

8. Nagel T (2001) Conceivableness of the impossibility and problem of the spirit and body. Voprosy Filosofii 8.

9. Sudakov KV (2010) Systemic mechanisms of mental activity. Zhurnal Nevrologii $i$ Psikhiatrii Imeni SS Korsakova 110: 4-14.

10. Popper K (2008) Knowledge and the body-mind problem: In defence of interaction. Translated from English by Zhuravlev IV, Moscow, pp: 256

11. Crick F, Koch C (1995) Why neuroscience may be able to explain consciousness. Scientific American 73: 84-85.

12. Sperry RW (1952) Neurology and the mind-brain problem. American Scientist 40: 291312 .

13. Yumatov EA (2013) Mental activity of the brain as a key to knowledge. Vestnik Mezhdunarodnoy Akademii Nauk 1: 35-45.
14. Yumatov EA (2014) To knowledge of the origin of the brain mental activity. World Journal of Neuroscience 4: 18213.

15. Yumatov EA (2019) Distance-field manifestations of brain mental activity. Biomeditsinskaya Radioelektronika 1: 5013

16. Grubov VV, Sitnikova E, Koronovskiy AA, Pavlov AN, Khramov AE (2012) Automatic isolation and analysis of oscillatory patterns in nonstationary signals of EEG by the wavelet transform and empirical mode method. Seriya Fizicheskaya 76: 1520-1523.

17. Koronovskiy AA, Makarov VA, Pavlov AN, Sitnikova E, Khramov AE (2013) Wavelets in neurodynamics and neurophysiology. Moscow.

18. Pavlov AN, Khramov AE, Koronovskiy AA, Sitnikova E, Makarov VA, Ovchinnikov AA (2012) Wavelet analysis in neurodynamics. Uspekhi Fiziologicheskikh Nauk 182 905-939.

19. Hramov AE, Koronovskii AA, Makarov VA, Pavlov AN, Sitnikova E (2015) Wavelets in neuroscience. Springer Heidelberg New York Dordrecht London, ISBN: 978-3-66243849-7, pp: 318 .

20. Yumatov EA, Khramov AE, Grubov VV, Glazachev OS, Dudnik EN, et al. (2018) Studying the possibility for recognition of brain mental activity by electroencephalogram wavelet analysis. Biomeditsinskaya Radioelektronika 4: 3-12.

21. Yumatov EA, Hramov AE, Grubov VV, Glazachev OS, Dudnik EN, et al. (2019) Possibility for recognition of psychic brain activity with continuous wavelet analysis of EEG. Journal of Behavioral and Brain Science 9.

22. MacDonald M (2012) Pro WPF 4.5 in C\#: Windows presentation foundation in NET 4.5 Apress, 4th edition, pp: 1095.

Copyright: ( 2019 Yumatov EA. This is an open-access article distributed under the terms of the Creative Commons Attribution License, which permits unrestricted use, distribution, and reproduction in any medium, provided the original author and source are credited. 